\title{
Article
}

\section{Study of asymptotic behavior of solutions of neutral mixed type difference equations}

\author{
Manel Gouasmia ${ }^{1}$, Abdelouaheb Ardjouni ${ }^{2, *}$ and Ahcene Djoudi ${ }^{1}$ \\ 1 Applied Mathematics Lab, Faculty of Sciences, Department of Mathematics, Univ Annaba, P.O. Box 12, Annaba \\ 23000, Algeria.; manelgouasmia3@gmail.com (M.G); adjoudi@yahoo.com (A.D) \\ 2 Faculty of Sciences and Technology, Department of Mathematics and Informatics, Univ Souk Ahras, P.O. Box 1553, \\ Souk Ahras, 41000, Algeria.
}

Received: 12 February 2020; Accepted: 1 March 2020; Published: 30 March $2020 .$.

\begin{abstract}
In this paper, we consider a neutral mixed type difference equation, and obtain explicitly sufficient conditions for asymptotic behavior of solutions. A necessary condition is provided as well. An example is given to illustrate our main results.
\end{abstract}

Keywords: Contraction mapping, neutral difference equations, mixed type, asymptotic behavior.

MSC: 34K20, 34K30, 34D04.

\section{Introduction}

C ertainly, the Lyapunov direct method has been, for more than 100 years, the efficient tool for the study of stability properties of ordinary, functional, partial differential and difference equations. Nevertheless, the application of this method to problems of stability in differential and difference equations with delay has encountered serious difficulties if the delay is unbounded or if the equation has unbounded terms ([1-16]). Recently, Burton, Furumochi, Zhang, Raffoul, Islam, Yankson and others have noticed that some of these difficulties vanish or might be overcome by means of fixed point theory (see [17-32]). The fixed point theory does not only solve the problem on stability but has a significant advantage over Lyapunov's direct method. The conditions of the former are often averages but those of the latter are usually pointwise (see [1]).

In this paper, we consider the following mixed type neutral difference equation

$$
\Delta x(t)+a(t) \Delta x(\tau(t))+\sum_{i=1}^{k} b_{i}(t) x\left(\sigma_{i}(t)\right)+\sum_{j=1}^{l} c_{j}(t) x\left(\tau_{j}(t)\right)=0,
$$

with an assumed initial condition

$$
x(t)=\psi(t) \text { for } t \in\left[m\left(t_{0}\right), t_{0}\right] \cap \mathbb{Z},
$$

where $\psi:\left[m\left(t_{0}\right), t_{0}\right] \cap \mathbb{Z} \rightarrow \mathbb{R}$ is a bounded sequence and for $t_{0} \geq 0$

$$
m\left(t_{0}\right)=\inf \left\{\sigma_{i}(s): s \geq t_{0}, i=1, \ldots k\right\} .
$$

Here $\Delta$ denotes the forward difference operator $\Delta x(t)=x(t+1)-x(t)$ for any sequence $\left\{x(t), t \in \mathbb{Z}^{+}\right\}$. For more details on the calculus of difference equations, we refer the reader to [11] and [24]. Throughout this paper, we assume that $a, b_{i}$ and $c_{j}$ are bounded sequences, and $\tau, \sigma_{i}$ and $\tau_{j}$ are non-negative sequences such that

$$
\begin{aligned}
\tau(t) & \rightarrow \infty \text { as } t \rightarrow \infty, \tau(t) \geq t, t \geq t_{0}, \\
\sigma_{i}(t) & \rightarrow \infty \text { as } t \rightarrow \infty, i=1, \ldots, k, \sigma_{i}(t) \leq t, t \geq t_{0} \\
\tau_{j}(t) & \rightarrow \infty \text { as } t \rightarrow \infty, j=1, \ldots, l, \tau_{j}(t) \geq t, t \geq t_{0} .
\end{aligned}
$$


Equation (1) can be viewed as a discrete analogue of the mixed type neutral differential equation;

$$
x^{\prime}(t)+a(t) x^{\prime}(\tau(t))+\sum_{i=1}^{k} b_{i}(t) x\left(\sigma_{i}(t)\right)+\sum_{j=1}^{l} c_{j}(t) x\left(\tau_{j}(t)\right)=0
$$

In [25], Bicer investigated (3) and obtained the asymptotic behavior of solutions. Our purpose here is to show the asymptotic behavior of solutions for (1). An asymptotic stability theorem with a necessary and sufficient condition is proved by using the contraction mapping theorem. For details on contraction mapping principle we refer the reader to [33]. An example is given to illustrate our main results.

\section{Main results}

Theorem 1. Let $a, b_{i}$ and $c_{j}$ non positive sequences. Assume that the following inequality has a nonnegative solution

$$
-a(t) \lambda(\tau(t)) \prod_{u=t}^{\tau(t)-1}(1-\lambda(u))-\sum_{i=1}^{k} b_{i}(t) \prod_{u=t}^{\sigma_{i}(t)-1}(1-\lambda(u))-\sum_{j=1}^{l} c_{j}(t) \prod_{u=t}^{\tau_{j}(t)-1}(1-\lambda(u)) \leq \lambda(t), t \geq t_{0}
$$

with $\lambda(t)<1$. Then, (1) has a positive solution.

Proof. Let $\lambda_{0}$ be a nonnegative solution of (1). Set

$$
\lambda_{n}(t)=\left\{\begin{array}{l}
\lambda_{n-1}(t), \quad \text { if } m\left(t_{0}\right) \leq t \leq t_{0} \\
-a(t) \lambda_{n-1}(\tau(t)) \prod_{u=t}^{\tau(t)-1}\left(1-\lambda_{n-1}(u)\right)-\sum_{i=1}^{k} b_{i}(t) \prod_{u=t}^{\sigma_{i}(t)-1}\left(1-\lambda_{n-1}(u)\right) \\
-\sum_{j=1}^{l} c_{j}(t) \prod_{u=t}^{\tau_{j}(t)-1}\left(1-\lambda_{n-1}(u)\right), t \geq t_{0}
\end{array}\right.
$$

for $n=1,2, \ldots$. Then, by (1), we get

$\lambda_{0}(t) \geq-a(t) \lambda_{0}(\tau(t)) \prod_{u=t}^{\tau(t)-1}\left(1-\lambda_{0}(u)\right)-\sum_{i=1}^{k} b_{i}(t) \prod_{u=t}^{\sigma_{i}(t)-1}\left(1-\lambda_{0}(u)\right)-\sum_{j=1}^{l} c_{j}(t) \prod_{u=t}^{\tau_{j}(t)-1}\left(1-\lambda_{0}(u)\right)=\lambda_{1}(t)$.

Then, we obtain $\lambda_{0}(t) \geq \lambda_{1}(t) \geq \ldots \geq \lambda_{n}(t) \geq 0$. So, there exists a pointwise $\operatorname{limit} \lambda(t)=\lim _{n \rightarrow \infty} \lambda_{n}(t)$. So, from the Lebesgue convergence theorem, we obtain

$$
\lambda(t)=-a(t) \lambda(\tau(t)) \prod_{u=t}^{\tau(t)-1}(1-\lambda(u))-\sum_{i=1}^{k} b_{i}(t) \prod_{u=t}^{\sigma_{i}(t)-1}(1-\lambda(u))-\sum_{j=1}^{l} c_{j}(t) \prod_{u=t}^{\tau_{j}(t)-1}(1-\lambda(u))
$$

Hence,

$$
x(t)=\left\{\begin{array}{l}
\lambda(t), \quad \text { if } m\left(t_{0}\right) \leq t \leq t_{0} \\
\lambda\left(t_{0}\right) \prod_{u=t_{0}}^{t-1}(1-\lambda(u)), t \geq t_{0}
\end{array}\right.
$$

is a positive solution of (1).

Theorem 2. Let $a, b_{i}$ and $c_{j}$ be non positive sequences and let $\Delta a(t)>0, a\left(t_{0}\right) \neq-\infty$. If

$$
\sum_{u=t_{0}}^{\infty} \sum_{j=1}^{l} c_{j}(u)=-\infty,
$$

and $x$ is a eventually positive solution of (1), then $x(t) \rightarrow \infty$ as $t \rightarrow \infty$. 
Proof. Assume that $x(t)>0$ for $t \geq T_{1}$. Choose $T \geq T_{1}$ such that $T_{1} \leq \inf \left\{\sigma_{i}(s): s \geq T, i=1, \ldots, k\right\}$. Then $\Delta x(t)+a(t) \Delta x(\tau(t)) \geq 0$, for $t \geq T$,

$$
\Delta x(t)+a(t) \Delta x(\tau(t))=-\sum_{i=1}^{k} b_{i}(t) x\left(\sigma_{i}(t)\right)-\sum_{j=1}^{l} c_{j}(t) x\left(\tau_{j}(t)\right),
$$

and

$$
\Delta[a(t) x(\tau(t))]=a(t) \Delta x(\tau(t))+\Delta a(t) x(\tau(t+1))
$$

that is

$$
\Delta[x(t)+a(t) x(\tau(t))]-\Delta a(t) x(\tau(t+1)) \geq-\sum_{j=1}^{l} c_{j}(t) x\left(\tau_{j}(t)\right) .
$$

From this, we can write

$$
\Delta[x(t)+a(t) x(\tau(t))] \geq-\sum_{j=1}^{l} c_{j}(t) x\left(\tau_{j}(t)\right),
$$

so

$$
\Delta[x(t)+a(t) x(\tau(t))] \geq-x(T) \sum_{j=1}^{l} c_{j}(t),
$$

which implies

$$
x(t)+a(t) x(\tau(t)) \geq a\left(t_{0}\right) x\left(\tau\left(t_{0}\right)\right)-x(T) \sum_{u=t_{0}}^{t-1} \sum_{j=1}^{l} c_{j}(u)
$$

So, we get

$$
x(t) \geq a\left(t_{0}\right) x\left(\tau\left(t_{0}\right)\right)-x(T) \sum_{u=t_{0}}^{t-1} \sum_{j=1}^{l} c_{j}(u) .
$$

Then $x(t) \rightarrow \infty$ as $t \rightarrow \infty$.

Theorem 3. Let $a(t)>0, b_{i}$ and $c_{j}$ be nonnegative sequences and let $\Delta a(t)<0, a\left(t_{0}\right) \neq \infty$. If

$$
\sum_{u=t_{0}}^{\infty} \sum_{j=1}^{l} c_{j}(u)=\infty
$$

and $x$ is a eventually positive solution of (1), then $x(t) \rightarrow 0$ as $t \rightarrow \infty$.

Proof. For $t \geq T_{1}$, since $x(t)>0$ we Choose $T \geq T_{1}$ such that $T_{1} \leq \inf \left\{\sigma_{i}(s): s \geq T, i=1, \ldots, k\right\}$. Then $\Delta x(t)+a(t) \Delta x(\tau(t)) \leq 0$, for $t \geq T$, and

$$
\Delta x(t)+a(t) \Delta x(\tau(t)) \leq-\sum_{j=1}^{l} c_{j}(t) x\left(\tau_{j}(t)\right),
$$

that is

$$
\Delta[x(t)+a(t) x(\tau(t))]-\Delta a(t) x(\tau(t+1)) \leq-\sum_{j=1}^{l} c_{j}(t) x\left(\tau_{j}(t)\right) .
$$

From this, we can write

$$
\Delta[x(t)+a(t) x(\tau(t))] \leq-\sum_{j=1}^{l} c_{j}(t) x\left(\tau_{j}(t)\right),
$$


so

$$
\Delta[x(t)+a(t) x(\tau(t))] \leq-x(T) \sum_{j=1}^{l} c_{j}(t)
$$

which implies

$$
x(t)+a(t) x(\tau(t)) \leq a\left(t_{0}\right) x\left(\tau\left(t_{0}\right)\right)-x(T) \sum_{u=t_{0}}^{t-1} \sum_{j=1}^{l} c_{j}(u) .
$$

So, we get

$$
x(t) \leq a\left(t_{0}\right) x\left(\tau\left(t_{0}\right)\right)-x(T) \sum_{u=t_{0}}^{t-1} \sum_{j=1}^{l} c_{j}(u) .
$$

Since $x(t)>0$, we get a contradiction. Then $x(t) \rightarrow 0$ as $t \rightarrow \infty$.

Now, we investigate the asymptotic behavior of solutions of (1), free of the sign of the coefficients. During the process of inverting (1), an summation by parts will have to performed on the term involving $\Delta x(\tau(t))$.

Lemma 1. A sequence $x$ is a solution of (1)-(2) if and only if

$$
\begin{aligned}
x(t)= & \left(x\left(t_{0}\right)+a\left(t_{0}-1\right) x\left(\tau\left(t_{0}\right)\right)\right) \prod_{s=t_{0}}^{t-1}(1-B(s))-a(t-1) x(\tau(t)) \\
& +\sum_{r=t_{0}}^{t-1} \prod_{s=r+1}^{t-1}(1-B(s)) h(r) x(\tau(r))-\sum_{r=t_{0}}^{t-1} \prod_{s=r+1}^{t-1}(1-B(s)) B(r) x(r+1) \\
& -\sum_{r=t_{0}}^{t-1} \prod_{s=r+1}^{t-1}(1-B(s)) \sum_{i=1}^{k} b_{i}(r) x\left(\sigma_{i}(r)\right)-\sum_{r=t_{0}}^{t-1} \prod_{s=r+1}^{t-1}(1-B(s)) \sum_{j=1}^{l} c_{j}(r) x\left(\tau_{j}(r)\right),
\end{aligned}
$$

for $t \geq t_{0}$, where

$$
B(t)=\sum_{i=1}^{k} b_{i}(t)+\sum_{j=1}^{l} c_{j}(t), 0<B(t)<1,
$$

and

$$
h(t)=a(t)-a(t-1)(1-B(t))
$$

Proof. Since

$$
x\left(\tau_{j}(t)\right)=x(t+1)+\sum_{u=t+1}^{\tau_{j}(t)-1} \Delta x(u), \text { and } x\left(\sigma_{i}(t)\right)=x(t+1)+\sum_{u=t+1}^{\sigma_{i}(t)-1} \Delta x(u) .
$$

We can rewrite (1) as

$$
\Delta x(t)=-a(t) \Delta x(\tau(t))-\sum_{i=1}^{k} b_{i}(t) \sum_{u=t+1}^{\sigma_{i}(t)-1} \Delta x(u)-\sum_{j=1}^{l} c_{j}(t) \sum_{u=t+1}^{\tau_{j}(t)-1} \Delta x(u)-B(t) x(t+1) .
$$

Multiplying both sides of (6) with $\prod_{s=t_{0}}^{t}(1-B(s))^{-1}$, by summing from $t_{0}$ to $t-1$, we obtain

$$
\begin{aligned}
& \sum_{r=t_{0}}^{t-1} \Delta\left[\prod_{s=t_{0}}^{r-1}(1-B(s))^{-1} x(r)\right]=-\sum_{r=t_{0}}^{t-1} \prod_{s=t_{0}}^{r}(1-B(s))^{-1} a(r) \Delta x(\tau(r))-\sum_{r=t_{0}}^{t-1} \prod_{s=t_{0}}^{r}(1-B(s))^{-1} B(r) x(r+1) \\
& -\sum_{r=t_{0}}^{t-1} \prod_{s=t_{0}}^{r}(1-B(s))^{-1} \sum_{i=1}^{k} b_{i}(t) \sum_{u=t+1}^{\sigma_{i}(r)-1} \Delta x(u)-\sum_{r=t_{0}}^{t-1} \prod_{s=t_{0}}^{r}(1-B(s))^{-1} \sum_{j=1}^{l} c_{j}(t) \sum_{u=t+1}^{\tau_{j}(r)} \Delta x(u) .
\end{aligned}
$$


By dividing both sides of the above expression by $\prod_{s=t_{0}}^{t-1}(1-B(s))^{-1}$ we get

$$
\begin{aligned}
x(t)= & x\left(t_{0}\right) \prod_{s=t_{0}}^{t-1}(1-B(s))-\sum_{r=t_{0}}^{t-1} \prod_{s=r+1}^{t-1}(1-B(s)) a(r) \Delta x(\tau(r))-\sum_{r=t_{0}}^{t-1} \prod_{s=r+1}^{t-1}(1-B(s)) B(r) x(r+1) \\
& -\sum_{r=t_{0}}^{t-1} \prod_{s=r+1}^{t-1}(1-B(s)) \sum_{i=1}^{k} b_{i}(r) \sum_{u=t+1}^{\sigma_{i}(r)-1} \Delta x(u)-\sum_{r=t_{0}}^{t-1} \prod_{s=r+1}^{t-1}(1-B(s)) \sum_{j=1}^{l} c_{j}(r) \sum_{u=t+1}^{\tau_{j}(r)-1} \Delta x(u) .
\end{aligned}
$$

By performing an summation by parts, we get

$$
\begin{aligned}
& \sum_{r=t_{0}}^{t-1} \prod_{s=r+1}^{t-1}(1-B(s)) a(r) \Delta x(\tau(r)) \\
& =a(t-1) x(\tau(t))-a\left(t_{0}-1\right) x\left(\tau\left(t_{0}\right)\right) \prod_{s=t_{0}}^{t-1}(1-B(s))-\sum_{r=t_{0}}^{t-1} \Delta\left[\prod_{s=r}^{t-1}(1-B(s)) a(r-1)\right] x(\tau(r)) .
\end{aligned}
$$

But,

$$
\begin{aligned}
\sum_{r=t_{0}}^{t-1} \Delta\left[\prod_{s=r}^{t-1}(1-B(s)) a(r-1)\right] x(\tau(r))=\sum_{r=t_{0}}^{t-1} \prod_{s=r+1}^{t-1}(1-B(s))[a(r)-a(r-1)(1-B(r))] x(\tau(r)) & \\
& =\sum_{r=t_{0}}^{t-1} \prod_{s=r+1}^{t-1}(1-B(s)) h
\end{aligned}
$$

where $h$ is given by (5). We obtain (4) by replacing (8) into (7). Since each step is reversible, the converse follows easily. This completes the proof.

Theorem 4. Assume that $0<B(t)<1$ and the following conditions hold

$$
\prod_{s=t_{0}}^{t-1}(1-B(s)) \rightarrow 0 \text { as } t \rightarrow \infty
$$

and

$$
\begin{aligned}
& |a(t-1)|+\sum_{r=t_{0}}^{t-1} \prod_{s=r+1}^{t-1}(1-B(s))|h(r)|+\sum_{r=t_{0}}^{t-1} \prod_{s=r+1}^{t-1}(1-B(s))|B(r)| \\
& +\sum_{r=t_{0}}^{t-1} \prod_{s=r+1}^{t-1}(1-B(s)) \sum_{i=1}^{k}\left|b_{i}(r)\right|+\sum_{r=t_{0}}^{t-1} \prod_{s=r+1}^{t-1}(1-B(s)) \sum_{j=1}^{l}\left|c_{j}(r)\right| \leq \beta<1 .
\end{aligned}
$$

Then for each initial condition (2), every solution of (1) converges to zero.

Proof. Let $x \in C\left(\left[m\left(t_{0}\right), \infty\right) \cap \mathbb{Z}\right)$ is the space of all bounded sequences and $M=\left\{x \in C\left(\left[m\left(t_{0}\right), \infty\right) \cap\right.\right.$ $\mathbb{Z}): x(t) \rightarrow 0$ as $t \rightarrow \infty\}$, be a closed subspace. Then $(M,\|\cdot\|)$ is a Banach space with the norm $\|x\|=$ $\sup _{t \geq m\left(t_{0}\right)}|x(t)|$.

Define the operator $\phi: M \rightarrow M$ by

$$
(\phi x)(t)=\left\{\begin{array}{l}
\psi(t), \quad \text { if } m\left(t_{0}\right) \leq t \leq t_{0}, \\
\left(x\left(t_{0}\right)+a\left(t_{0}-1\right) x\left(\tau\left(t_{0}\right)\right)\right) \prod_{s=t_{0}}^{t-1}(1-B(s))-a(t-1) x(\tau(t)) \\
+\sum_{r=t_{0}}^{t-1} \prod_{s=r+1}^{t-1}(1-B(s)) h(r) x(\tau(r))-\sum_{r=t_{0}}^{t-1} \prod_{s=r+1}^{t-1}(1-B(s)) B(r) x(r+1) \\
-\sum_{r=t_{0}}^{t-1} \prod_{s=r+1}^{t-1}(1-B(s)) \sum_{i=1}^{k} b_{i}(r) x\left(\sigma_{i}(r)\right)-\sum_{r=t_{0}}^{t-1} \prod_{s=r+1}^{t-1}(1-B(s)) \sum_{j=1}^{l} c_{j}(r) x\left(\tau_{j}(r)\right), t \geq t_{0} .
\end{array}\right.
$$


It is clear that for $x \in M, \phi x$ is bounded. Now, we will show that $\phi$ is a contraction. Let $x$ and $y$ be two bounded sequences on $\left[m\left(t_{0}\right), \infty\right) \cap \mathbb{Z}$ and satisfying same initial condition (2). Then for $t \geq t_{0}$, we get

$$
\begin{aligned}
& |(\phi x)(t)-(\phi y)(t)| \leq|a(t-1)||x(\tau(t))-y(\tau(t))|+\sum_{r=t_{0}}^{t-1} \prod_{s=r+1}^{t-1}(1-B(s))|h(r)||x(\tau(r))-y(\tau(r))| \\
& +\sum_{r=t_{0}}^{t-1} \prod_{s=r+1}^{t-1}(1-B(s))|x(r+1)-y(r+1)||B(r)|+\sum_{r=t_{0}}^{t-1} \prod_{s=r+1}^{t-1}(1-B(s)) \sum_{i=1}^{k}\left|b_{i}(r)\right|\left|x\left(\sigma_{i}(r)\right)-y\left(\sigma_{i}(r)\right)\right| \\
& +\sum_{r=t_{0}}^{t-1} \prod_{s=r+1}^{t-1}(1-B(s)) \sum_{j=1}^{l}\left|c_{j}(r)\right|\left|x\left(\tau_{j}(r)\right)-y\left(\tau_{j}(r)\right)\right| \leq \beta\|x-y\| .
\end{aligned}
$$

Thus, the operator $\phi$ has a unique fixed point in $M$, which solves (1). Now, we will show that, $(\phi x)(t) \rightarrow 0$ as $t \rightarrow \infty$. Actually, for $x \in M$, we have

$$
\begin{aligned}
|(\phi x)(t)| \leq & \left|\left(x\left(t_{0}\right)+a\left(t_{0}-1\right) x\left(\tau\left(t_{0}\right)\right)\right)\right| \prod_{s=t_{0}}^{t-1}(1-B(s))+|a(t-1)||x(\tau(t))| \\
& +\sum_{r=t_{0}}^{t-1} \prod_{s=r+1}^{t-1}(1-B(s))|h(r)||x(\tau(r))|+\sum_{r=t_{0}}^{t-1} \prod_{s=r+1}^{t-1}(1-B(s))|x(r+1)||B(r)| \\
& +\sum_{r=t_{0}}^{t-1} \prod_{s=r+1}^{t-1}(1-B(s)) \sum_{i=1}^{k}\left|b_{i}(r)\right|\left|x\left(\sigma_{i}(r)\right)\right|+\sum_{r=t_{0}}^{t-1} \prod_{s=r+1}^{t-1}(1-B(s)) \sum_{j=1}^{l}\left|c_{j}(r)\right|\left|x\left(\tau_{j}(r)\right)\right| .
\end{aligned}
$$

Note that by (9),

$$
\left|\left(x\left(t_{0}\right)+a\left(t_{0}-1\right) x\left(\tau\left(t_{0}\right)\right)\right)\right| \prod_{s=t_{0}}^{t-1}(1-B(s)) \rightarrow 0 \text { as } t \rightarrow \infty .
$$

Moreover, since $x(t) \rightarrow 0$ as $t \rightarrow \infty$, for each $\varepsilon>0$, there exists $T_{1}>t_{0}$ such that $u \geq T_{1}$ implies that $|x(\tau(u))|<\frac{\varepsilon}{2}$. Thus, for $t \geq T_{1}$, the third term $I_{3}$ in (12) satisfies

$$
\begin{aligned}
I_{3} & =\sum_{r=t_{0}}^{t-1} \prod_{s=r+1}^{t-1}(1-B(s))|h(r)||x(\tau(r))| \\
& \leq \sum_{r=t_{0}}^{T_{1}-1} \prod_{s=r+1}^{t-1}(1-B(s))|h(r)||x(\tau(r))|+\sum_{r=T_{1}}^{t-1} \sum_{r=t_{0}}^{t-1} \prod_{s=r+1}^{t-1}(1-B(s))|h(r)||x(\tau(r))| \\
& \leq \sum_{r=t_{0}}^{T_{1}-1} \sum_{r=t_{0}}^{t-1} \prod_{s=r+1}^{t-1}(1-B(s))|h(r)||x(\tau(r))|+\frac{\varepsilon}{2} \sum_{r=T_{1}}^{t-1} \sum_{r=t_{0}}^{t-1} \prod_{s=r+1}^{t-1}(1-B(s))|h(r)| \\
& \leq \frac{\varepsilon}{2}+\beta \frac{\varepsilon}{2} \\
& \leq \varepsilon
\end{aligned}
$$

Thus $I_{3} \rightarrow 0$ as $t \rightarrow \infty$. By a similar technique, we can prove that the rest of terms in (12) tend zero as $t \rightarrow \infty$. Therefore $(\phi x)(t) \rightarrow 0$ as $t \rightarrow \infty$. This completes the proof.

Theorem 5. Suppose that $0<B(t)<1$. If all solutions of (1) converge to zero, then (9) holds.

Proof. Suppose that (9) does not holds. That is,

$$
\lim _{t \rightarrow \infty} \prod_{s=t_{0}}^{t-1}(1-B(s))=\delta \neq 0
$$


So, from (13), we can write $\delta \neq 0$. Then, there exists a sequence $\left\{t_{n}\right\}$ approaching $\infty$, such that

$$
\prod_{s=t_{0}}^{t_{n}-1}(1-B(s)) \rightarrow \delta \text { as } n \rightarrow \infty .
$$

For $x\left(t_{0}\right) \neq 0$, let $x$ be a solution. Then,

$$
\lim _{n \rightarrow \infty}\left|\left(x\left(t_{0}\right)+a\left(t_{0}-1\right) x\left(\tau\left(t_{0}\right)\right)\right)\right| \prod_{s=t_{0}}^{t_{n}-1}(1-B(s))=\left|\left(x\left(t_{0}\right)+a\left(t_{0}-1\right) x\left(\tau\left(t_{0}\right)\right)\right)\right| \delta \neq 0 .
$$

From Lemma 1, $x\left(t_{n}\right)$ satisfies (4). On the other hand, we know that

$$
\begin{aligned}
& \lim _{n \rightarrow \infty}\left[\sum_{r=t_{0}}^{t_{n}-1} \prod_{s=r+1}^{t_{n}-1}(1-B(s))|h(r)||x(\tau(r))|-\left|a\left(t_{n}-1\right)\right|\left|x\left(\tau\left(t_{n}\right)\right)\right|\right. \\
& +\sum_{r=t_{0}}^{t_{n}-1} \prod_{s=r+1}^{t_{n}-1}(1-B(s))|x(r+1)||B(r)|+\sum_{r=t_{0}}^{t_{n}-1} \prod_{s=r+1}^{t_{n}-1}(1-B(s)) \sum_{i=1}^{k}\left|b_{i}(r)\right|\left|x\left(\sigma_{i}(r)\right)\right| \\
& \left.+\sum_{r=t_{0}}^{t_{n}-1} \prod_{s=r+1}^{t_{n}-1}(1-B(s)) \sum_{j=1}^{l}\left|c_{j}(r)\right|\left|x\left(\tau_{j}(r)\right)\right|\right]=0 .
\end{aligned}
$$

Since all solutions tend zero, from (4), (14) and (15), we get

$$
\lim _{n \rightarrow \infty} x\left(t_{n}\right)=\left|\left(x\left(t_{0}\right)+a\left(t_{0}-1\right) x\left(\tau\left(t_{0}\right)\right)\right)\right| \delta \neq 0,
$$

which contradicts all solutions of (1) converge to zero. The proof is completed.

We end the paper with the following example.

Example 1. consider the mixed type neutral difference equation

$$
\Delta x(t)+a(t) \Delta x(\tau(t))+b_{1}(t) x\left(\sigma_{1}(t)\right)+c_{1}(t) x\left(\tau_{1}(t)\right)=0,
$$

with an assumed initial condition

$$
x(t)=\psi(t) \text { for } t \in\left[m\left(t_{0}\right), t_{0}\right] \cap \mathbb{Z},
$$

where $t_{0}=0, m\left(t_{0}\right)=-2, \psi(t)=t / 3, a(t)=\frac{1}{3^{t+2}}, b_{1}(t)=1-\frac{1}{2^{t}}, c_{1}(t)=\frac{1}{2^{t+1}}, \tau(t)=3 t / 2, \sigma_{1}(t)=$ $t / 2-2, \tau_{1}(t)=5 t / 2$. We have

$$
B(t)=1-\frac{1}{2^{t+1}}, \prod_{s=0}^{t-1}(1-B(s))=\prod_{s=0}^{t-1} \frac{1}{2^{s+1}} \rightarrow 0 \text { as } t \rightarrow \infty,
$$

and

$$
\begin{aligned}
& |a(t-1)|+\sum_{r=t_{0}}^{t-1} \prod_{s=r+1}^{t-1}(1-B(s))|h(r)|+\sum_{r=t_{0}}^{t-1} \prod_{s=r+1}^{t-1}(1-B(s))|B(r)| \\
& +\sum_{r=t_{0}}^{t-1} \prod_{s=r+1}^{t-1}(1-B(s))\left|b_{1}(r)\right|+\sum_{r=0}^{t-1} \prod_{s=r+1}^{t-1}(1-B(s))\left|c_{1}(r)\right| \\
& =\frac{1}{3^{t+1}}+\sum_{r=0}^{t-1} \prod_{s=r+1}^{t-1} \frac{1}{2^{s+1}}\left|\frac{1}{3^{r+2}}-\frac{1}{3^{r+1} \times 2^{r+1}}\right|+\sum_{r=0}^{t-1} \prod_{s=r+1}^{t-1} \frac{1}{2^{s+1}}\left(1-\frac{1}{2^{r+1}}\right) \\
& +\sum_{r=0}^{t-1} \prod_{s=r+1}^{t-1} \frac{1}{2^{s+1}}\left(1-\frac{1}{2^{r}}\right)+\sum_{r=0}^{t-1} \prod_{s=r+1}^{t-1} \frac{1}{2^{s+1}} \times \frac{1}{2^{r+1}} \simeq 0.722<1 .
\end{aligned}
$$


Thus all the conditions of Theorem 4 are satisfied and every solution of (16) converges to zero.

\section{Concluding remarks}

In this article, a neutral mixed type difference equation is considered. The asymptotic behavior of solutions is obtained with a necessary and sufficient condition by using fixed point theorems. The results are supported with a suitable illustrative example.

Author Contributions: All authors contributed equally to the writing of this paper. All authors read and approved the final manuscript.

Conflicts of Interest: "The authors declare no conflict of interest."

\section{References}

[1] Burton, T. A. (2013). Stability by fixed point theory for functional differential equations. Courier Corporation, New York.

[2] Burton, T. A., \& Furumochi, T. (2001). Fixed points and problems in stability theory for ordinary and functional differential equations. Dynamic Systems and Applications, 10(1), 89-116.

[3] Ardjouni, A., \& Djoudi, A. (2012). Fixed points and stability in nonlinear neutral differential equations with variable delays. Nonlinear Studies, 19(3), 345-357.

[4] Ardjouni, A., \& Djoudi, A. (2011). Stability in nonlinear neutral differential equations with variable delays using fixed point theory. Electronic Journal of Qualitative Theory of Differential Equations, 2011(43), 1-11.

[5] Ardjouni, A., Derrardjia, I., \& Djoudi, A. (2014). Stability in totally nonlinear neutral differential equations with variable delay. Acta Mathematica Universitatis Comenianae, 83(1), 119-134.

[6] Ardjouni, A., Djoudi, A., \& Soualhia, I. (2012). Stability for linear neutral integro-differential equations with variable delays. Electronic journal of Differential Equations, 172(2012), 1-14.

[7] Becker, L. C., \& Burton, T. A. (2006). Stability, fixed points and inverses of delays. Proceedings of the Royal Society of Edinburgh Section A: Mathematics, 136(2), 245-275.

[8] Derrardjia, I., Ardjouni, A., \& Djoudi, A. (2013). Stability by Krasnoselskii's theorem in totally nonlinear neutral differential equations. Opuscula Mathematica, 33(2), 255-272.

[9] Dung, N. T. (2015). Asymptotic behavior of linear advanced differential equations. Acta Mathematica Scientia, 35(3), 610-618.

[10] Dung, N. T. (2013). New stability conditions for mixed linear Levin-Nohel integro-differential equations. Journal of Mathematical Physics, 54(8), 082705.

[11] Elaydi, S. (1999). An Introduction to Difference Equations, Springer, New York.

[12] Elaydi, S. (1994). Periodicity and stability of linear Volterra difference systems. Journal of Mathematical Analysis and Applications, 181(2), 483-492.

[13] Elaydi, S., \& Murakami, S. (1997). Uniform asymptoic stability in linear volterra difference equations. Journal of Difference Equations and Applications, 3(3-4), 203-218.

[14] Eloe, P. W., Islam, M. N., \& Raffoul, Y. N. (2003). Uniform asymptotic stability in nonlinear Volterra discrete systems. Computers \& Mathematics with Applications, 45(6-9), 1033-1039.

[15] Islam, M. N., \& Raffoul, Y. N. (2003). Exponential Stability in Non-linear Difference Equations. Journal of Difference Equations and Applications, 9(9), 819-825.

[16] Raffoul, Y. N. (2003). General theorems for stability and boundedness for nonlinear functional discrete systems. Journal of mathematical analysis and applications, 279(2), 639-650.

[17] Ardjouni, A., \& Djoudi, A. (2015). Stability in nonlinear neutral difference equations. Afrika Matematika, 26(3-4), 559-574.

[18] Ardjouni, A., \& Djoudi, A. (2015). Asymptotic stability in totally nonlinear neutral difference equations. Proyecciones (Antofagasta), 34(3), 255-276.

[19] Ardjouni, A., \& Djoudi, A. (2014). Stability in nonlinear neutral integro-differential equations with variable delay using fixed point theory. Journal of Applied Mathematics and Computing, 44(1-2), 317-336.

[20] Ardjouni, A., \& Djoudi, A. (2013). Stability in linear neutral difference equations with variable delays. Mathematica Bohemica, 138(3), 245-258.

[21] Ardjouni, A., \& Djoudi, A. (2013). Stability in nonlinear neutral difference equations with variable delays. TJMM, 5(1), 01-10.

[22] Ardjouni, A., \& Djoudi, A. (2012). Fixed points and stability in neutral nonlinear differential equations with variable delays. Opuscula Mathematica, 32(1), 5-19. 
[23] Islam, M., \& Yankson, E. (2005). Boundedness and stability in nonlinear delay difference equations employing fixed point theory. Electronic Journal of Qualitative Theory of Differential Equations, 2005(26), 1-18.

[24] Kelley, W. G., \& Peterson, A. C. (2001). Difference equations: an introduction with applications. Academic press.

[25] Bicer, E. (2018). On the Asymptotic Behavior of Solutions of Neutral Mixed Type Differential Equations. Results in Mathematics, 73(4), 1-12.

[26] Mesmouli, M. B., Ardjouni, A., \& Djoudi, A. (2014). Study of the stability in nonlinear neutral differential equations with functional delay using KrasnoselskiiÜBurtonŠs fixed-point. Applied Mathematics and Computation, 243, $492-502$.

[27] Mesmouli, M. B., Ardjouni, A., \& Djoudi, A. (2014). Stability in neutral nonlinear differential equations with functional delay using Krasnoselskii-Burton's fixed-point. Nonlinear Studies, 21(4), 601-617.

[28] Raffoul, Y. N. (2006). Stability and periodicity in discrete delay equations. Journal of mathematical analysis and applications, 324(2), 1356-1362.

[29] Raffoul, Y. N. (2004). Periodicity in general delay non-linear difference equations using fixed point theory. Journal of Difference Equations and Applications, 10(13-15), 1229-1242.

[30] Yankson, E. (2009). Stability in discrete equations with variable delays. Electronic Journal of Qualitative Theory of Differential Equations, 2009(8), 1-7.

[31] Yankson, E. (2006). Stability of Volterra difference delay equations. Electronic Journal of Qualitative Theory of Differential Equations, 2006(20), 1-14.

[32] Zhang, B. (2005). Fixed points and stability in differential equations with variable delays. Nonlinear Analysis: Theory, Methods E Applications, 63(5-7), e233-e242.

[33] Smart, D. R. (1974). Fixed point theorems, Cambridge Tracts in Mathematics. Cambridge University Press, London-New York.

(C) 2020 by the author; licensee PSRP, Lahore, Pakistan. This article is an open access article distributed under the terms and conditions of the Creative Commons Attribution (CC-BY) license (http://creativecommons.org/licenses/by/4.0/). 